\title{
Realignment of Liquid Crystal Shells Driven by Temperature- Dependent Surfactant Solubility
}

\author{
Anjali Sharma, Venkata Subba Rao Jampani, ${ }^{(0}$ and Jan P. F. Lagerwall*(;) \\ University of Luxembourg, 162a, Avenue de la Faiencerie, 1511 Luxembourg, Grand Duchy of Luxembourg
}

Supporting Information

ABSTRACT: We investigate dynamic director field variations in shells of the nematic liquid crystal (LC) compound, 4-cyano-4' pentylbiphenyl, suspended in and containing immiscible aqueous phases. The outer and inner shell interfaces are stabilized by the cationic surfactant, cetyl trimethyl ammonium bromide (CTAB), and by the water soluble polymer, poly(vinyl alcohol) (PVA), respectively. PVA and surfactant solutions normally promote tangential and orthogonal alignments, respectively, of the LC director. The rather high Krafft temperature of $\mathrm{CTAB}, \mathrm{T}_{\mathrm{K}} \approx$ $25{ }^{\circ} \mathrm{C}$, means that its solubility in water is below the critical

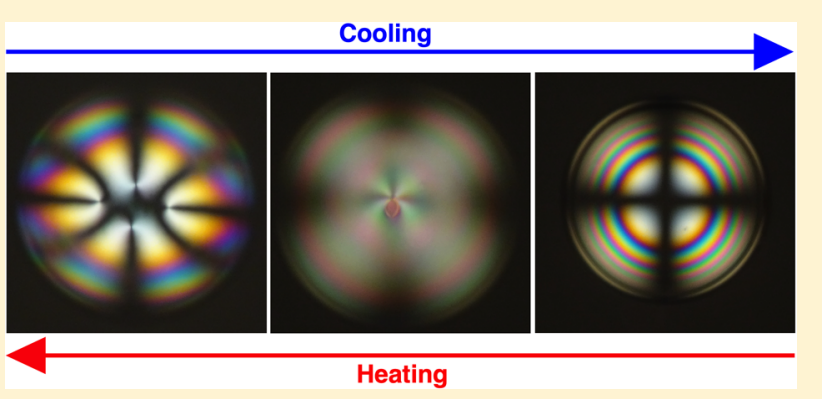
micelle concentration at room temperature in most labs. Here, we study the effect of cooling/heating past $T_{\mathrm{K}}$ on the LC shell director configuration. Within a certain concentration range, $\mathrm{CTAB}$ in the outer aqueous phase (and PVA in the inner) switches the LC director field from hybrid to uniformly orthogonal upon cooling below $T_{\mathrm{K}}$. We argue that the effect is related to the migration of the surfactant through the fluid LC membrane into the initially surfactant-free aqueous PVA solution, triggered by the drastically reduced water solubility of CTAB at $T<T_{\mathrm{K}}$. The results suggest that LC shells can detect solutes in the continuous phase, provided there is sufficient probability that the solute migrates through the LC into the inner aqueous phase.

\section{INTRODUCTION}

Nematic liquid crystals (LCs) are ordered fluids, in which the anisometric molecules (mesogens, typically rod- or discshaped) exhibit long-range orientational order along the socalled director, $\hat{n}{ }^{1}$ The long-range orientational order means that the physical properties are different along and perpendicular to $\hat{n}$, hence controlling its orientation is critical. Conversely, because the optical properties-in particular, the direction of the optic axis of a birefringent LC-depend on $\hat{n}$, a polarizing microscopic study of LC textures can reveal geometrical variations of the director field. As the director configuration is typically set by choosing appropriate boundary conditions, the LC can effectively work as a detector or amplifier of molecular-scale events that influence these boundary conditions. $^{2}$

The situation of an LC in contact with an immiscible liquid is interesting, thanks to the freedom of the interface to curve. LCs confined with a curved geometry can develop a variety of director field configurations with corresponding intriguing textures. Particularly rich is the case where the LC is suspended in, and also contains a droplet of, water or another immiscible liquid, i.e., the LC forms a self-closing shell. ${ }^{3,4}$ By varying the type of LC, a plethora of fascinating phenomena can be induced and studied, stimulating from a fundamental science point of view ${ }^{5-15}$ as well as from an application perspective. ${ }^{16-25}$ In all of these cases, the director alignment at the inand outside of the shell is key to the behavior displayed by the
LC; hence, controlling the LC alignment at each interface has great practical significance.

A schematic representation of a shell is shown in Figure 1a, where the inner blue sphere and the blue background represent the inner droplet and the outer continuous phase (normally both aqueous solutions), respectively, and the yellow sphere represents the LC phase. Typically, the shell thickness is a few microns, whereas the diameter is on the order of $100 \mu \mathrm{m}$. For the LC to remain in the form of a shell, it must be immiscible with both surrounding phases. At the same time, the interfacial tension between the LC and its bounding phases must be kept sufficiently low, as otherwise the emulsification into shells becomes prohibitively difficult. Low interfacial tension also extends the shell lifetime, as does kinetic stabilization, preventing close encounters of shells that could otherwise lead to coalescence into an LC droplet. We achieve the required reduced interfacial tension and/or kinetic stabilization by adding a suitable polymer or a surfactant as a stabilizer, ${ }^{26}$ typically mixed into the aqueous phases. The choice of stabilizer is critical also from the perspective of tuning the LC director field, as the stabilizer may have a direct impact on the orientation of $\hat{n}$ at the interface. ${ }^{27,28}$

Direct contact of the LC with pure water promotes alignment with $\hat{n}$ in the interface plane (tangential align-

Received: April 4, 2019

Revised: July 12, 2019

Published: July 29, 2019 

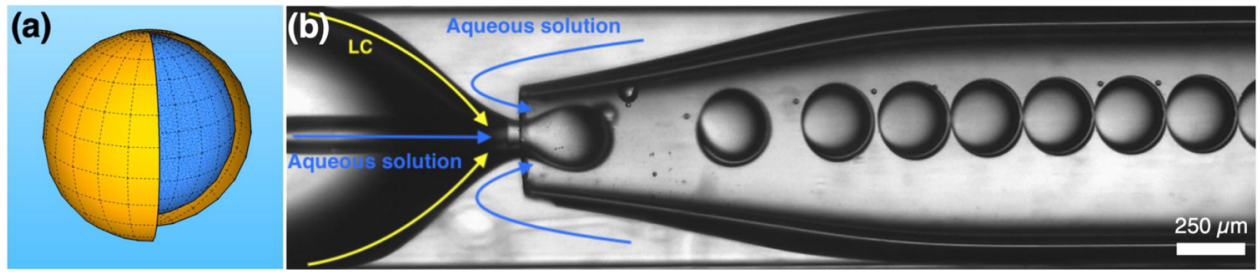

Figure 1. (a) Schematic of the structure of a shell, with the LC drawn in yellow and the inner aqueous droplet in blue. (b) Snapshot of shell production where blue arrows represent the inner and outer aqueous phases and yellow arrows show the LC flowing as a middle phase.

ment), ${ }^{29,30}$ remaining also if a polymeric stabilizer such as poly(vinyl alcohol) (PVA) is dissolved in the water. $^{28}$ In contrast, dissolution of low-molar-mass surfactants in the aqueous phase is generally assumed to give an orthogonal alignment, ${ }^{29,30}$ as the surfactant tends to adsorb at the interface with an orthogonal orientation. However, in a recent systematic study of LC shells stabilized by anionic or cationic surfactants, with varying length of the hydrophobic tail, we found that multiple director configurations can, in fact, be stabilized with surfactants alone, by carefully choosing the surfactant type and concentration. ${ }^{27}$ One of the cationic surfactants in that study, cetyl (hexadecyl) trimethyl ammonium bromide (CTAB), is commonly used in colloid science but has so far not been employed much for stabilizing LC shells.

$\mathrm{CTAB}$ is interesting as it has a Krafft temperature near room temperature, $T_{\mathrm{K}} \approx 25{ }^{\circ} \mathrm{C}$. This makes it potentially useful for dynamically tuning the alignment of LC shells, as we may expect very different surfactant-LC interactions for temperatures $T<T_{\mathrm{K}}$, at least if the solution has an overall CTAB content greater than the critical micelle concentration (CMC). This is because the water solubility of an ionic surfactant reduces to below the CMC at $T<T_{\mathrm{K}}$, rendering micelle formation unfavorable. In the absence of other liquids, excess surfactant will then either nucleate as crystals or condense onto available interfaces. This phenomenon has previously been used to disperse nanoparticles with optimal surfactant coverage $^{31}$ and it is, thus, worthwhile studying what the effects are at the much larger scale of a liquid crystal shell. Here, the surfactant adsorption at interfaces is not only important for reducing the interfacial tension and avoiding aggregation, ${ }^{32}$ but it also influences the director alignment, hence changing surfactant-LC interactions should be recognized in changes to the LC shell texture. Moreover, in contrast to surfactant-based dispersion of solid particles, here the additional freedom of surfactant penetration into or even through the interface must also be considered, with potentially interesting consequences.

These are the issues we explore in this paper, using the standard room-temperature nematic-forming mesogen, 4cyano-4'-pentylbiphenyl (5CB), for the LC shell. We show that for a certain range of $C T A B$ concentrations in the outer aqueous phase the LC director configuration in a shell can be switched conveniently by heating or cooling past the Krafft temperature. With PVA in the inner phase, the LC shell is hybrid-aligned at $T>T_{\mathrm{K}}$, as expected, but if we cool to $T<T_{\mathrm{K}}$ the LC uniformly adopts orthogonal $\hat{n}$ throughout the shell. However, the option of the surfactant to solubilize LC into droplets, a process that is greatly promoted by cooling toward or below $T_{\mathrm{K}}$, has critical consequences for the shell lifetime and the overall character of the suspension. We present the results with different concentrations of $\mathrm{CTAB}$, we compare the effects of having the surfactant on the outside or on the inside of the shell, and we also study the response of the LC shell when a surrounding aqueous solution contains both PVA and CTAB dissolved together.

\section{EXPERIMENTAL SECTION}

Materials. CTAB ( $\geq 98 \%$ purity, Carl Roth), PVA (Sigma-Aldrich, $M_{\mathrm{w}}=13-23 \mathrm{~kg} / \mathrm{mol}, 87-89 \%$ hydrolyzed), and 5CB (>99\% purity, Yantai Xianhua Chem-Tech) were used as received without further purification. All solutions were prepared by dissolving the surfactant in deionized water (resistivity $18 \mathrm{M} \Omega / \mathrm{cm}$, Sartorius arium pro DI) and stirring at $40{ }^{\circ} \mathrm{C}$ for 1 day.

Shell Production. LC shells containing and surrounded by aqueous phases containing different concentrations of $\mathrm{CTAB}$ and PVA were produced by using a coaxial glass capillary microfluidic setup, as described by Weitz and co-workers. ${ }^{33}$ An inner aqueous solution, immiscible with the middle phase (LC), is flown through a tapered cylindrical capillary (inlet) with a $70 \mu \mathrm{m}$ diameter orifice, injected into the LC that is flowing in the same direction. The LC and the inner aqueous phase were flow-focused by a counter-flowing aqueous phase to encapsulate the LC between the two aqueous phases, as shown in Figure 1b. All shells were produced at $T>T_{\mathrm{K}}$ for $\mathrm{CTAB}$ by keeping the coaxial capillary setup on a tailor-made hot stage. The resulting emulsion was collected in a glass vial containing the same solution as that used as the outer fluid in production, via an outlet capillary with an orifice diameter of $300 \mu \mathrm{m}$. Within minutes, a sample of the emulsion was transferred from the vial into rectangular capillaries, the ends of which were sealed by high-temperature glue to avoid evaporation of water. Each capillary containing shells was placed in a Linkam T95-PE hot stage mounted on a Nikon Eclipse LV100ND polarizing optical microscope (POM). Although pure 5CB has a melting point of $24{ }^{\circ} \mathrm{C}$, it supercools over long times, allowing us to study the shells at significantly lower temperature, without crystallization over the time frame of the experiment. Videos and images were captured by a Sony FDR AX33 camcorder, mounted on the microscope.

Conductivity Measurements. We prepared aqueous $\mathrm{CTAB}$ solutions of $0.12,0.25,0.5,1.0,3.0,5.0,7.0,9.0,11.0,13.0$, and 15.0 $\mathrm{mM}$ concentrations. CTAB + PVA solutions of the same CTAB concentrations were also prepared, with the PVA concentration fixed at 5 wt \%. To measure the conductivity (using a Eutech Instrument CON 450 conductometer), we filled aqueous solutions in glass vials, keeping them on a heating plate to ensure that all measurements are conducted at the same temperature $\left(33.8{ }^{\circ} \mathrm{C}\right)$.

After measuring the conductivities of pure $\mathrm{CTAB}$ and $\mathrm{CTAB}+$ PVA solutions as a reference dataset, we prepared shell suspensions using a $15 \mathrm{mM} \mathrm{CTAB}$ solution in the outer phase and $5 \mathrm{wt} \%$ PVA solution in the inner phase. We collected the suspension in a $40 \mathrm{~mL}$ glass vial and kept it at $30^{\circ} \mathrm{C}$ to prevent CTAB crystallization. Due to density mismatch, the shells sediment to the bottom of the vial, allowing us to extract an aliquot of the outer phase into a $10 \mathrm{~mL}$ vial for measuring its conductivity. We kept the remaining suspension at $3{ }^{\circ} \mathrm{C}$ for almost $10 \mathrm{~min}$ and then extracted aliquots of the outer phase twice more, first at $3{ }^{\circ} \mathrm{C}$ and then after having heated the suspension to $30{ }^{\circ} \mathrm{C}$. Because the volume extracted at $3{ }^{\circ} \mathrm{C}$ was only $2 \mathrm{~mL}$, which was too small for carrying out the conductivity measurement, we diluted this phase to $10 \mathrm{~mL}$ by adding deionized water. To establish 


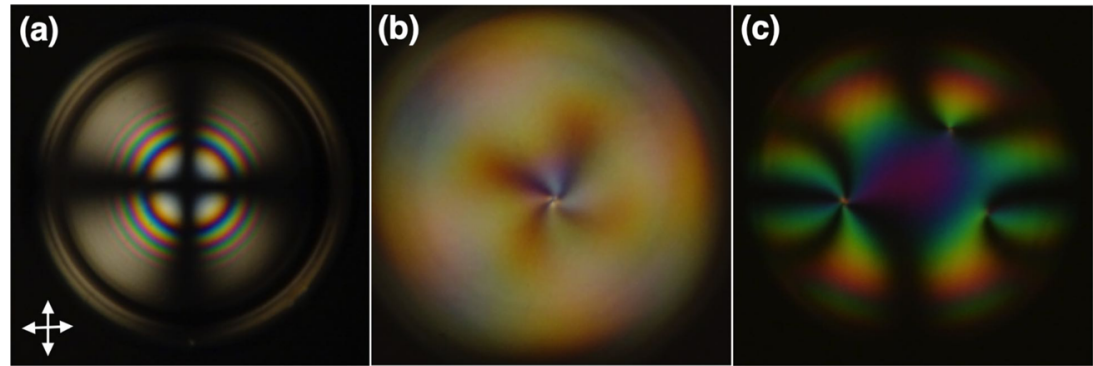

Figure 2. POM images of $5 \mathrm{CB}$ shells containing and suspended in aqueous solutions, containing different stabilizers. (a) An orthogonally aligned shell stabilized by $0.5 \mathrm{mM} \mathrm{CTAB}$ in the inner and outer phases, (b) a hybrid-aligned shell with $0.5 \mathrm{mM} \mathrm{CTAB}$ in the inner phase and 5 wt \% PVA in the outer phase, and (c) a tangentially aligned shell with $5 \mathrm{wt} \%$ PVA in both aqueous phases. Crossed arrows indicate the polarizer and analyzer orientations.

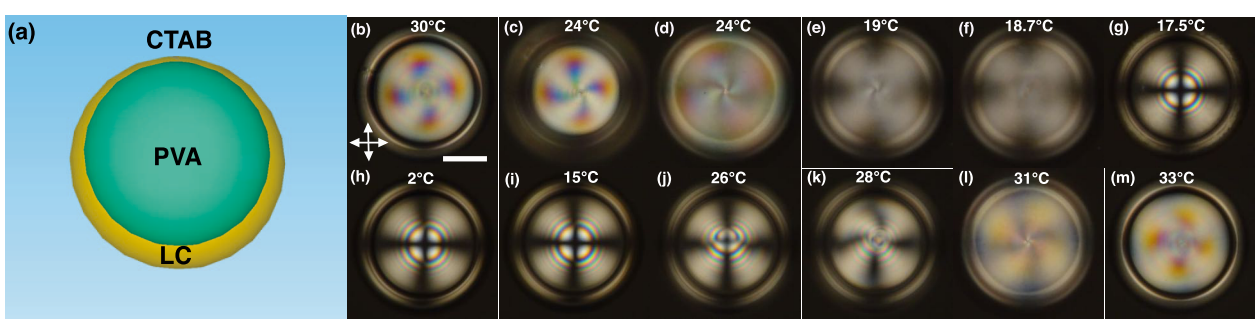

Figure 3. (a) Schematic side view of a $5 \mathrm{CB}$ shell with $\mathrm{CTAB}$ in the outer and PVA in the inner aqueous phase. The remaining panels show POM images (top view) of the change in the nematic shell alignment with temperature for an outer-phase CTAB concentration of $4 \mathrm{mM}$; (b-h) hybrid to orthogonal alignment change on cooling from 30 to $1{ }^{\circ} \mathrm{C}$; $(\mathrm{i}-\mathrm{m})$ change from orthogonal to hybrid on heating back to $33{ }^{\circ} \mathrm{C}$. The photo in panel (c) is focused on the bottom part of the shell, photos in (e), (f), (k), and (l) are focused on the top part, whereas the rest are focused on the equator. Crossed arrows indicate the polarizer and analyzer orientations, and the scale bar represents $50 \mu \mathrm{m}$.

the conductivity, and corresponding $\mathrm{CTAB}$ concentration, of the original phase, the dilution was taken into account as described later.

\section{RESULTS}

As a reference, we first produce shells with temperatureindependent director fields, avoiding surfactant concentrations above CMC, which is $0.9 \mathrm{mM}$ for CTAB. With $0.5 \mathrm{mM}$ aqueous $\mathrm{CTAB}$ solution in the inner as well as the outer phase, the CTAB aligns the LC orthogonally to the boundaries on both interfaces. ${ }^{27}$ These shells thus display a characteristic orthogonal alignment texture, ${ }^{34}$ as shown in Figure $2 \mathrm{a}$. If we replace one of the CTAB solutions with a 5 wt \% PVA aqueous solution, either on the inside or on the outside, the shell adopts a hybrid director field, going from tangential at one interface to orthogonal at the other. This is recognized through the typical texture of hybrid-aligned shells, ${ }^{13,35}$ with a +1 topological defect at the shell top and another at the bottom, as in Figure 2b. If both aqueous phases are PVA solutions, finally, then the LC is tangentially aligned at both the LC-water interfaces. Shells with such fully tangentially aligned director fields frequently develop more than two defects, including either two or four $+1 / 2$ defects, ${ }^{14,15}$ as in Figure $2 c$.

CTAB Solutions above the CMC as the Outer Phase. We now produce shells with systematically increasing concentration of $\mathrm{CTAB}$, specifically $0.5,2,4,6,10,15$, and $20 \mathrm{mM}$, in the continuous outer phase, while maintaining a 5 wt \% aqueous PVA solution as the inner phase. As illustrated in Figure $3 \mathrm{a}$, the shell is slightly thinner at the top than at the bottom, since the aqueous phase has somewhat lower density than 5CB. For each CTAB concentration, we study the alignment of the 5CB in the shells as a function of temperature, at a cooling/heating rate of $2{ }^{\circ} \mathrm{C} / \mathrm{min}$. We cool the emulsion from the isotropic phase of $5 \mathrm{CB}$ (the clearing point is $35^{\circ} \mathrm{C}$ ) to $1{ }^{\circ} \mathrm{C}$ and then heat it back until the shell again turns isotropic.

For the lowest CTAB concentrations, the shells are hybridaligned as expected by the imposed boundary conditions, and the alignment does not change with temperature; see Figure S3 in the Supporting Information. Interestingly, on increasing the concentration of $\mathrm{CTAB}$ above the $\mathrm{CMC}$, we observe a reversible change in alignment when passing the Krafft temperature. On cooling, the alignment changes to orthogonal at $T<T_{\mathrm{K}}$, as seen in Figure 3, showing a shell where $4 \mathrm{mM}$ $\mathrm{CTAB}$ is in the outer phase. At $18.7^{\circ} \mathrm{C}$ (Figure 3f), we observe that the +1 defect at the top of the shell vanishes, and the alignment changes to uniformly orthogonal (Figure $3 g$ ). On heating, the orthogonal texture starts showing distortions around $T_{\mathrm{K}}$ (panel $\mathrm{j}$ in Figure 3), and the alignment gradually changes back to hybrid (panels $1-\mathrm{m}$ ). We may again recognize the +1 defect at the top of the shell in Figure 31 .

A further increase in $\mathrm{CTAB}$ concentration shows the same transition from hybrid to orthogonal up to $10 \mathrm{mM} \mathrm{CTAB}$. At $15 \mathrm{mM}$ CTAB, we observe the same change in alignment on first cooling, at $24{ }^{\circ} \mathrm{C}$ (Figure S4d), but this time the alignment does not revert to hybrid when we heat. Even after heating the shells to the isotropic phase and then cooling back, the alignment remains orthogonal. If we continue to increase the CTAB concentration to $20 \mathrm{mM}$, we never see the hybrid alignment but obtain permanently orthogonally aligned shells regardless of temperature.

Most experiments are conducted on shells with a thickness at the shell equator of about $10 \mu \mathrm{m}$, but we also explore thinner as well as thicker shells. The thinnest shells that can be produced with a reasonable lifetime are about $8 \mu \mathrm{m}$ thick at the equator, and they respond as described above. The behavior remains unchanged also for thicker shells up to $35 \mu \mathrm{m}$ 
equator thickness, whereas shells with an equator thickness of about $60 \mu \mathrm{m}$ do not change their alignment upon cooling (Figure S10).

If we invert the geometry of the shell, placing the $\mathrm{CTAB}$ solution on the inside and the PVA solution on the outside, we must increase the concentration of CTAB further to see the impact of the Krafft temperature. For $30 \mathrm{mM} \mathrm{CTAB}$ concentration, the alignment change from hybrid to orthogonal could be reproduced also with this geometry, whereas for lower surfactant concentrations the director field is hybrid regardless of temperature. Example textures are shown for the case of 15 and $30 \mathrm{mM} \mathrm{CTAB}$ in the inner aqueous solution in Figures S1 and S2.

The reversible change in alignment between hybrid and orthogonal thus occurs only for a range of CTAB concentrations, which is above the CMC and below $15 \mathrm{mM}$ when $C T A B$ is in the outside continuous phase, with a higher threshold concentration required for the reverse geometry. We do not attempt to identify the upper limit for the latter geometry, because a very high surfactant concentration, like what is required here, leads to rapid degradation of the shell, as explained in the Discussion section. We summarize the alignment transition temperatures for shells stabilized by different concentrations of CTAB in the continuous phase in Figure 4, observing a certain hysteresis between cooling and

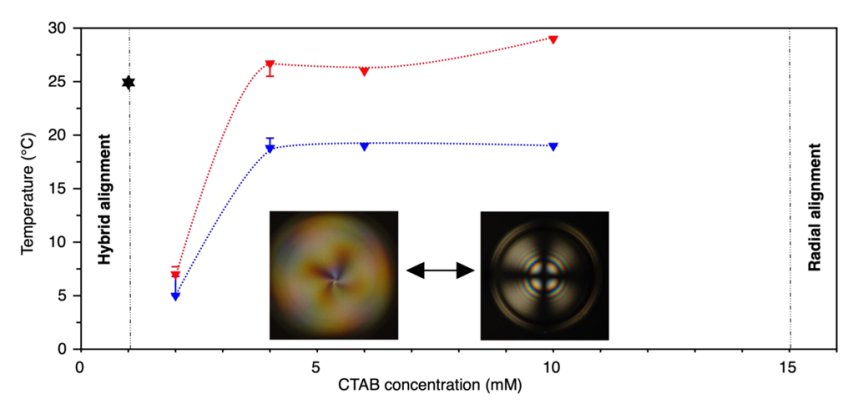

Figure 4. Approximate temperatures of the hybrid-to-orthogonal (blue, on cooling) and orthogonal-to-hybrid (red, on heating) transitions as a function of $\mathrm{CTAB}$ concentration. The star indicates $T_{\mathrm{K}}$ for CTAB. Lines are guides to the eye.

heating. Note that the transitions are not very sharp, and the indicated temperatures should be considered as approximate. For two CTAB concentrations, the experiment was conducted five times, allowing us to estimate an error for the temperature of onset of the alignment transition, as indicated with error bars for the corresponding data points. We also find that each transition temperature is fairly constant for $\mathrm{CTAB}$ concentrations 4,6 , and $10 \mathrm{mM}$, but at $2 \mathrm{mM} \mathrm{CTAB}$ concentration, both transitions are much lower, requiring cooling far below $T_{\mathrm{K}}$ to see the alignment transition.

PVA + CTAB Solution in the Outer Phase. For studying the aligning strengths of the two stabilizers when they are both present in the same phase, we also produce shells similar to the previous case but with the addition of PVA in the outer phase along with $\mathrm{CTAB}$, as shown schematically in Figure 5a. The concentration of PVA is fixed to the same value as in the CTAB-free inner phase, 5 wt \% as in the previous section, and we vary the CTAB concentration from 1 to $30 \mathrm{mM}$.

Interestingly, with 1 and $5 \mathrm{mM}$ solution of CTAB in PVA, shells are tangentially aligned regardless of temperature, i.e., the orthogonal-aligning influence of CTAB is counteracted by the presence of the PVA at these solute concentrations. It is noteworthy that already $0.5 \mathrm{mM} C \mathrm{CTAB}$ was sufficient to induce an orthogonally aligned interface in the absence of PVA; here we have 2-10 times higher concentration of CTAB, yet its influence is canceled out by the PVA in the same solution. To see the influence of CTAB on $\hat{n}$, we must increase its concentration in the PVA solution to $8 \mathrm{mM}$ and we must cool below $T_{K}$; see Figure $5 b-m$. We then see a transition from tangential to uniformly orthogonal via an intermediate transitory texture that is, however, distinctly different from the hybrid alignment. The process is reversible and follows the same sequence backward on heating to $30{ }^{\circ} \mathrm{C}$.

Increasing the $\mathrm{CTAB}$ concentration to $10 \mathrm{mM}$ still yields a tangential high-temperature state, but transitions are different from the shell with $8 \mathrm{mM}$ CTAB. At this surfactant concentration, we observe the characteristic hybrid alignment at intermediate temperatures instead of the transitory texture. On cooling further, the alignment changes from hybrid to uniformly orthogonal. However, now the shell does not recover the original tangential alignment on heating, as we observed in the previous case. The transition from orthogonal to hybrid still takes place around $16-20{ }^{\circ} \mathrm{C}$, but on further heating the shell remains in the hybrid configuration; see Figure S5.

With a further increase in $\mathrm{CTAB}$ concentration from 15 to $30 \mathrm{mM}$, we observe that the shell is hybrid-aligned even at $30{ }^{\circ} \mathrm{C}$, instead of tangential as we observed in the $10 \mathrm{mM}$ case. On cooling, the alignment still changes from hybrid to uniformly orthogonal, as shown in Figure S6 and, on heating, it changes back to hybrid at $25^{\circ} \mathrm{C}$.

We can identify three different concentration regions; see Figure 6. For $\mathrm{CTAB}$ concentrations $c_{\mathrm{CTAB}}$ in the range 0$5 \mathrm{mM}$, the shell is tangentially aligned at all temperatures. For
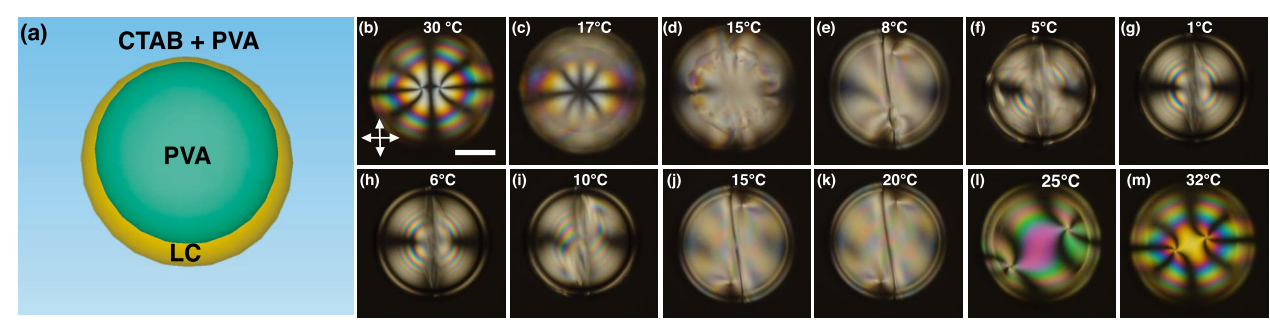

Figure 5. (a) Schematic side view of a shell with CTAB + PVA in the outer aqueous phase and aqueous PVA solution in the inner phase. The remaining panels show POM images (top view) of the change in the nematic 5CB shell alignment with temperature for an outer phase containing 5 wt \% PVA and $8 \mathrm{mM} \mathrm{CTAB}$. Tangential alignment is seen at $30^{\circ} \mathrm{C}(\mathrm{b})$. On cooling, the shell starts changing alignment at $17^{\circ} \mathrm{C}(\mathrm{c})$, passing through a transitory texture $(\mathrm{d}-\mathrm{f})$, before it becomes uniformly orthogonal at around $1{ }^{\circ} \mathrm{C}$. On heating, the alignment reverts, via the transitory state $(j-k)$, to tangential $(1-\mathrm{m})$. Crossed arrows indicate the polarizer and analyzer orientations, and the scale bar represents $50 \mu \mathrm{m}$. 


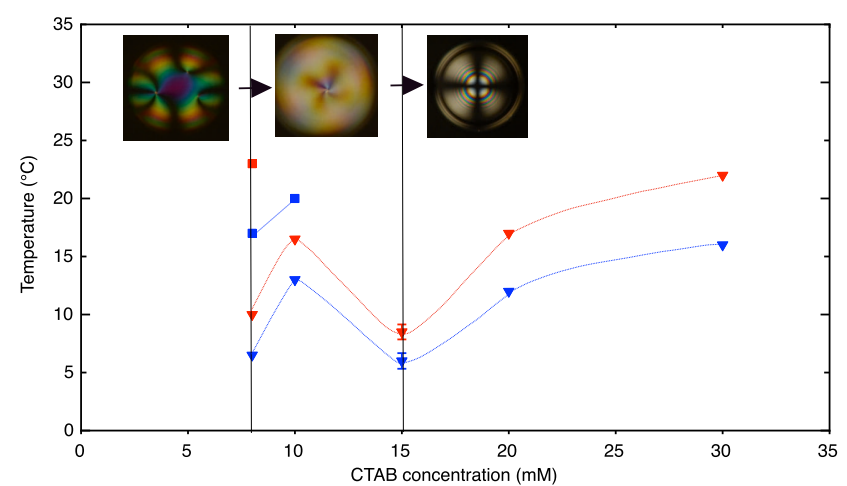

Figure 6. Tangential to hybrid to orthogonal switching temperatures (approximate) as a function of CTAB concentration, when both PVA and $\mathrm{CTAB}$ are present in the outer phase. In the first concentration regime, there is no change in alignment with temperature and the shell is tangentially aligned. In the second regime, we observe tangential to hybrid to orthogonal alignment change on cooling and heating. Blue squares represent the transition from tangential to hybrid on cooling, while the red square shows the hybrid to tangential transition on heating. In the third regime, we observe only hybrid to orthogonal alignment change on cooling, and this is represented by blue triangles, whereas red triangles show switching on heating. Lines are guides to the eye.

$8 \mathrm{mM} \leq c_{\text {СТAB }}<15 \mathrm{mM}$, we see the richest variation, with shells starting out tangential at high temperatures, and then on cooling first changing to hybrid or other transitional states (blue squares) followed by a transition to uniformly orthogonal (blue triangles). The red data points are for the corresponding transitions on heating. For $15 \mathrm{mM} \leq c_{\mathrm{CTAB}} \leq$ $30 \mathrm{mM}$, we have a reversible transition between hybrid at high temperatures and uniformly orthogonal at low temperatures. A fully tangential alignment no longer develops at any temperature in this range of $c_{\mathrm{CTAB}}$.

The alignment transition temperatures generally increase with increasing concentration of $\mathrm{CTAB}$, similar to the case in the previous section, where only $\mathrm{CTAB}$ was in the outer phase. The exception is $15 \mathrm{mM} \mathrm{CTAB}$, for which we have a dip in realignment temperatures, which is surprising. We repeated the experiment at this $\mathrm{CTAB}$ concentration three times to rule out that the dip is an experimental artifact. The richer variation in texture compared to the case when CTAB is on its own in the outer phase may be related to this dip, because $15 \mathrm{mM}$ also corresponds to the lowest $\mathrm{CTAB}$ concentration for which a tangential director alignment is never seen; we will return to this issue in the Discussion section. At $8 \mathrm{mM}$, we have reversible tangential to uniformly orthogonal alignment transitions, whereas at $10 \mathrm{mM}$, the shells start out with tangential director fields, but once the configuration changes to hybrid on cooling, the tangential configuration is never recovered, even on reheating. The minimum in realignment temperature may thus be at the boundary between different sequences of realignment.

To confirm that the alignment change is really driven by the different behavior of CTAB above and below $T_{\mathrm{K}}$, and not due to variations in the elastic constants of the $\mathrm{LC}$ as the temperature is varied, we conduct a reference experiment with shells made from the broad temperature range nematic mixture E7; see Figure S7. The clearing transition of E7 starts at about $63{ }^{\circ} \mathrm{C}$ and crystallization is suppressed to well below room temperature, hence the variations of the LC properties are negligible in the temperature range of our experiments. Since it is not in our interest to repeat the full study with E7 as the shell material, we choose the relatively high CTAB concentration of $50 \mathrm{mM}$ in the outside aqueous phase, together with 5 wt \% PVA, to ensure that we have enough surfactant to drive the phenomenon. With an inner phase of 5 wt \% aqueous PVA solution, we indeed see the same alignment transition from hybrid to uniformly orthogonal upon cooling below $T_{\mathrm{K}}$. This confirms that it is the temperature responsiveness of the surfactant that gives rise to the alignment change.

Changes in Conductivity and Turbidity of the Continuous Phase. To have an independent test of the concentration of mobile $\mathrm{CTAB}$ in the aqueous phase at each temperature, we measure the conductivity of samples of continuous phase extracted from a macroscopic vial containing a shell suspension prepared with $15 \mathrm{mM} \mathrm{CTAB}$ in the outer phase and 5\% PVA in the inner phase. The first extraction (see the Experimental Section for full details) is carried out $5 \mathrm{~min}$ after shell production, with the sample at a temperature of $33.8{ }^{\circ} \mathrm{C}$. The second extraction is done after the vial is cooled to $3{ }^{\circ} \mathrm{C}$, thus well below $T_{\mathrm{K}}$, and kept at this temperature for $10 \mathrm{~min}$, long enough that an alignment transition within the shells is expected. The third extraction is done after the cooled sample has been reheated to $30{ }^{\circ} \mathrm{C}$, with the measurement done again at $33.8{ }^{\circ} \mathrm{C}$ for consistency. By comparing the respective conductivity values with a reference curve for $\mathrm{CTAB}$ solutions without shells (Figure 7), we can estimate the concentration of freely dissolved $\mathrm{CTAB}$ in the outer aqueous phase.

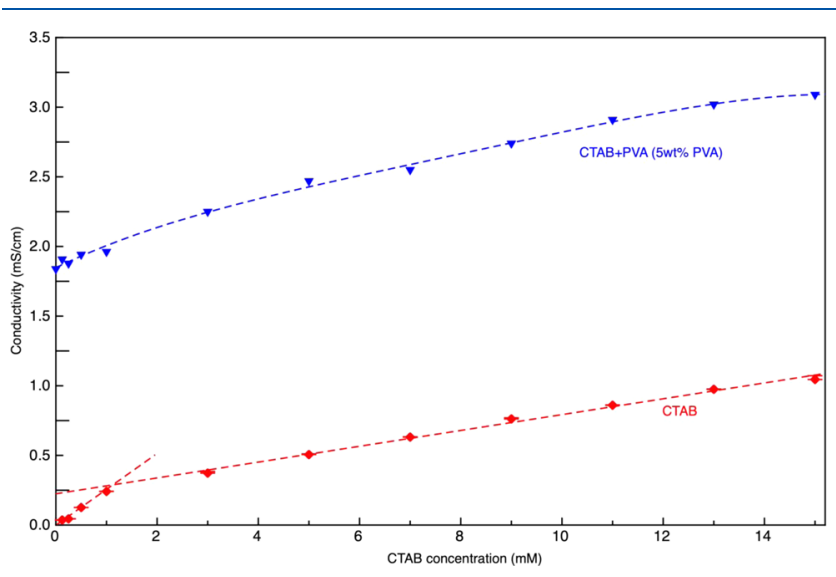

Figure 7. Conductivity of aqueous $\mathrm{CTAB}$ solutions, neat (red diamonds) and with 5\% PVA co-dissolved (blue triangles), as a function of CTAB concentration. The CMC can easily be identified in the neat solution as the concentration where the conductivity curve changes its slope, but when PVA is present no such feature is detectable. The dotted red lines are best linear fits to the red data points below and above $\mathrm{CMC}$, respectively, whereas the blue dotted line is a fourth-order polynomial fit to the blue data points.

The conductivity measured for the first extracted continuous phase, at $33.8{ }^{\circ} \mathrm{C}$ soon after shell production, is $712.3 \mu \mathrm{S} / \mathrm{cm}$ which, according to Figure 7, corresponds to a CTAB concentration of 8-9 $\mathrm{mM}$. The reduction in conductivity compared to that of a $15 \mathrm{mM} \mathrm{CTAB} \mathrm{solution} \mathrm{without} \mathrm{shells}$ can be explained by the fact that the shells constitute very large interfaces onto which $\mathrm{CTAB}$ adsorbs, limiting the mobilities of the $\mathrm{CTA}^{+}$surfactant molecules as well as its loosely bound $\mathrm{Br}^{-}$ counter ions to such an extent that they hardly contribute to 
the conductivity. In other words, we assume that the sample has 8-9 $\mathrm{mM}$ concentration of individually dissolved and micellar CTAB.

For condition 3, where the shell suspension has been kept at $3{ }^{\circ} \mathrm{C}$ for $10 \mathrm{~min}$ and then reheated to $30{ }^{\circ} \mathrm{C}$ prior to extraction, the measured conductivity is $604.3 \mu \mathrm{S} / \mathrm{cm}$. The reduced conductivity demonstrates that the cooling of the shell suspension below $T_{\mathrm{K}}$ leads to a reduction in freely dissolved $\mathrm{CTAB}$ even after reheating. Importantly, the continuous phase becomes turbid after the cooling experiment, as shown in Figure S8. A polarizing microscope investigation of a capillary subjected to a similar temperature history reveals that numerous small droplets of LC appear in the outer phase upon cooling the sample; see Figure S9. The significance of the conductivity drop and the turbidity due to LC droplet formation will be discussed below.

The conductivity corresponding to condition 2, finally, where the continuous phase is extracted at $3{ }^{\circ} \mathrm{C}$, was found to be $505.9 \mu \mathrm{S} / \mathrm{cm}$. This value is based on the conductivity of a diluted solution (see the Experimental Section), $0.241 \mathrm{mS} / \mathrm{cm}$, corresponding to a CTAB concentration of $1.0 \mathrm{mM}$, according to Figure 7. The established conductivity at condition 2 is significantly greater than the conductivity at the $\mathrm{CMC}$, in contrast to what would be expected for a pure aqueous $\mathrm{CTAB}$ solution cooled to below $T_{\mathrm{K}}$, since the solubility in water is lower than the CMC. Similar to the case of condition 3, the continuous phase extracted at $3{ }^{\circ} \mathrm{C}$ is turbid. When PVA is codissolved with $\mathrm{CTAB}$ in the continuous phase, cooling does not render the phase turbid, neither at $3{ }^{\circ} \mathrm{C}$ nor after reheating; see Figures S8 and S9.

\section{DISCUSSION}

The above results show that the director field configuration can be tuned dynamically in nematic LC shells, via variation of temperature over a convenient range, by combining suitable surfactants and polymer stabilizers in the surrounding aqueous phases. The question is what drives the alignment changessometimes reversible, sometimes permanent-between fully tangential, hybrid, and uniformly orthogonal, seen for certain $\mathrm{CTAB}$ concentration ranges as we cool below or heat above $T_{\mathrm{K}}$. Since $T_{\mathrm{K}}$ and CMC apparently play key roles, we need to consider the consequences of surfactant micellization and demicellization with respect to the action of CTAB on the LC shell, as well as how these processes are affected by the presence of PVA.

The reversible alignment change at intermediate $\mathrm{CTAB}$ concentrations in the outer phase would seem to suggest that we are seeing a pure adsorption/desorption phenomenon below and above $T_{\mathrm{K}}$. However, three key observations indicate that the situation is more complex. First, the temperaturedependent alignment is seen only when CTAB is on the outside for this range of concentrations, i.e., when the surfactant solution is the continuous phase, whereas the PVA solution is the disperse minority phase inside the shells. Second, the hybrid alignment at high temperatures is lost for $\mathrm{CTAB}$ concentrations in the outer phase greater than $10 \mathrm{mM}$ $\mathrm{CTAB}$; the shell director field is either orthogonal from the beginning or after the first cooling below $T_{K}$. Third, the reduced conductivity of the continuous phase after the sample has been cooled below $T_{\mathrm{K}}$ shows that the concentration of mobile $\mathrm{CTAB}$ in the continuous phase has been reduced by the cooling process in the presence of LC shells. For simplicity, the below discussion focuses on the case of CTAB in the continuous aqueous phase and PVA in the aqueous phase residing inside the shell.

We know from our earlier study of $5 \mathrm{CB}$ shells in contact with aqueous solutions of the surfactant, sodium dodecyl sulfate, that surfactant molecules can pass through the LC membrane from the inner to the outer phase or vice versa. ${ }^{28}$ Considering the longer alkyl chain (by four carbon atoms) of $\mathrm{CTAB}$ and the consequent higher solubility of $\mathrm{CTAB}$ in most LCs, the ability of CTAB to enter the LC is even greater. A transfer of surfactant to the aqueous phase on the other side is further facilitated by the asymmetry in shell thickness due to density mismatch between the LC and the inner aqueous phase, yielding a thinnest point of the shell, where transport of CTAB across the LC wall is particularly likely. We thus need to consider the possibility that the realignment is connected to $\mathrm{CTAB}$ passing through the LC and into the phase that initially contains no surfactant.

In aqueous surfactant solutions with a concentration above the $\mathrm{CMC}$-requiring that we are at temperatures $T>T_{\mathrm{K}}$, since otherwise the surfactant solubility is too low-the concentrations of individually dissolved surfactant molecules and of molecules adsorbed at interfaces remain essentially constant at the values reached at the $\mathrm{CMC}$, independent of the overall surfactant content. This is because additional surfactant molecules added beyond the CMC aggregate into micelles. If we cool such a solution to $T<T_{\mathrm{K}}$, the surfactant solubility in water reduces to less than the $\mathrm{CMC}$, and plain micelle formation is no longer favorable. Yet, the surfactant molecules that were in micelles remain. Hence, in a closed system, without an additional liquid phase, the only possible outcome is a meso- or macroscopic phase separation in which the excess surfactant molecules separate out of solution as solid crystals. ${ }^{31}$ Some surfactant molecules may aggregate at interfaces, which now become fully saturated, a small increase there being conceivable via a more ordered packing.

The slightly different situation for the case of our LC shell suspension is shown in the schematic drawing in Figure 8. In this case, the best available solvent for CTAB is reversed at $T<$ $T_{\mathrm{K}}$, meaning that the $\mathrm{LC}$ rather than the water can now act as a solvent, preventing the hydrophobic hexadecyl chains of CTAB from crystallizing. As the competition for interaction with the LC becomes strong at $T<T_{\mathrm{K}}$, the outer surfaces of the LC shells become supersaturated, and some $\mathrm{CTAB}$ may again disperse in the continuous phase as micelles, but this time they are much larger, filled with the LC extracted from the shell. ${ }^{36}$ We suspect that this happens primarily at points where the shell is thick, explaining the droplets (equivalent to LC-filled very large micelles) found in the continuous phase upon cooling the system, rendering the suspension turbid (Figure S8 and S9). At the thinnest part of the asymmetric shell, CTAB molecules may instead transfer to the inner aqueous core, which is not yet saturated with CTAB. In the process, they will tend to decorate the inside of the LC shell with the surfactant, promoting a director orientation that is orthogonal throughout the shell. However, they do so in competition with the PVA dissolved in this phase, an issue we will come back to below.

The transfer of CTAB from empty micelles in the continuous phase to the LC shell interior and the inclusion of LC into micelles that lose mobility due to their large increase in size explain the reduction in conductivity of the outer phase after having cooled the system down to $T<T_{\mathrm{K}}$. The LC-swollen micelles formed upon cooling also explain why the conductivity at $3{ }^{\circ} \mathrm{C}$ is more than twice as high as that 

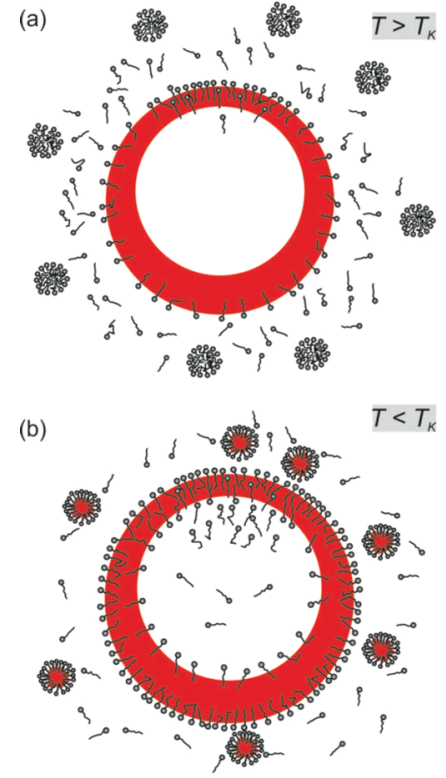

Figure 8. Schematic representation (not to scale) of the behavior of $\mathrm{CTAB}$ molecules in the outer aqueous solution at $c_{\mathrm{CTAB}}>\mathrm{CMC}$, in the vicinity of an LC shell at temperatures well above (a) and below (b) the Krafft temperature, $T<T_{\mathrm{K}}$. Case (a) describes the situation just after shell production, with the $\mathrm{CTAB}$ molecules in three different configurations: decorating the shell outside, aggregating into simple micelles, and individually dissolved in water. Cooling to $T<T_{\mathrm{K}}$ empty micelles are no longer stable, and the CTAB molecules that were in micelles therefore saturate the outer LC shell-water interface in addition to extracting the LC to form LC-filled swollen micelles (droplets) in the continuous phase. In addition, at the thinnest point of the asymmetric shell, the surfactant can easily move through the $\mathrm{LC}$ to the originally $\mathrm{CTAB}$-free aqueous phase on the shell interior. Since the inner phase also contains PVA (not depicted), the interaction between $\mathrm{CTAB}$ and PVA further complicates the behavior on the inside.

of an LC-free CTAB solution at the CMC: although the mobilities of these $\mathrm{CTA}^{+}$-solubilized LC droplets and their loosely bound $\mathrm{Br}^{-}$counter ions are lower than those of the empty micelles of the initial sample, they still contribute to the conductivity.

For the inner shell interface to receive sufficient CTAB for triggering the change of LC alignment from planar to orthogonal, the drive of $\mathrm{CTAB}$ from the continuous phase must be strong enough. At $c_{\mathrm{CTAB}}>4 \mathrm{mM}$ in the outer phase, this happens directly upon cooling below $T_{\mathrm{K}}$, but at $c_{\mathrm{CTAB}}=2$ $\mathrm{mM}$, the total surfactant concentration is apparently too close to the CMC for the effect to happen immediately upon passing $T_{\mathrm{K}}$. As seen in Figure 4, we thus have to continue cooling to a significantly lower temperature, inducing a yet lower $\mathrm{CTAB}$ solubility in the outer phase, to drive enough $\mathrm{CTAB}$ to the inside.

As the temperature is increased again, the water solubility increases above the $\mathrm{CMC}$, and $\mathrm{CTAB}$ can desorb, to some extent, from both interfaces, leaving the inner interface in contact mainly with PVA. This would explain the recovery of the hybrid alignment for low $\mathrm{CTAB}$ concentrations. With $c_{\mathrm{CTAB}} \geq 15 \mathrm{mM}$ in the continuous phase, the transport of surfactant molecules through the LC shell seems to be so strong that the shell remains orthogonal, either after a first cooling below $T_{\mathrm{K}}$ at $15 \mathrm{mM} \mathrm{CTAB}$, or from the very start, at any temperature, for higher concentrations.
The transport of CTAB across the LC membrane should take place also when we invert the shell geometry, with CTAB solution inside the shell to begin with. However, in this case, the $\mathrm{CTAB}$ molecules diffusing through the shell go from the minority internal phase to the vast continuous aqueous PVA solution in which the shells are suspended. This explains why a much greater $C T A B$ concentration is required to induce an alignment change with this geometry, as otherwise the flux of $\mathrm{CTAB}$ from the minority interior to the majority exterior phase will only induce a marginal presence of $\mathrm{CTAB}$ on the outside, insufficient to overpower the influence of PVA. Since the required high $\mathrm{CTAB}$ concentration, about 30 times the $\mathrm{CMC}$, leads to strong micellization of the LC, the shells change rapidly in behavior and have limited lifetime, rendering this geometry less interesting to study.

If our hypothesis that CTAB is transported through the LC shell membrane is correct, the second round of experiments, where PVA and CTAB are mixed together from the beginning, becomes highly interesting, since we would achieve this mixture of surfactant and polymer also in the initially pure PVA solution at $T<T_{\mathrm{K}}$. Particularly surprising is the fact that $\mathrm{CTAB}$ never succeeded in inducing the orthogonal alignment at either interface when present up to $5 \mathrm{mM}$ together with PVA. This may seem to contradict the observation in Figure 3, where the shell turned orthogonal below $T_{\mathrm{K}}$ although there was only $4 \mathrm{mM} \mathrm{CTAB}$ on the outside phase initially. Although we cannot measure the $\mathrm{CTAB}$ concentration on the inside after cooling, we know that it must be lower than $4 \mathrm{mM}$. And since the PVA concentration on the inside is the same $5 \mathrm{wt} \%$ as in the reference experiments with controlled PVA-CTAB mixture solutions, one might expect that no alignment change should be possible here either.

However, the process of driving CTAB through the LC phase constitutes a significant difference here, allowing better saturation of the LC-water interface compared to the case when $\mathrm{CTAB}$ is in the aqueous solution together with PVA from the start. The difference in behavior should be further enhanced by the direct PVA-CTAB interactions in aqueous solution, which will reduce the adsorption of surfactant at the LC-water interface in the second part of our study. In early studies of interactions between incompletely hydrolyzed PVA (as in our study) and CTAB in aqueous solution, ${ }^{37,38}$ it was demonstrated that the surfactant and polymer form rather strong complexes, sometimes called polymer-bound micelles. This is favored by the polymer's ability to reduce crowding of surfactant headgroups and water contact of the alkyl chains, as well as the surfactant's ability to cover the hydrophobic unhydrolyzed acetate pendants of the PVA. Moreover, the incompletely hydrolyzed PVA used in this study is generally regarded as being a lightly acidic solute, ${ }^{a}$ i.e., it tends to donate protons to the solution, leaving the polymer in aqueous solution with a weak negative charge. The $\mathrm{CTA}^{+}$ions are thus electrostatically attracted to the negatively charged deprotonated components of the PVA. The CTAB-PVA complexation means that less surfactant is available for interacting with the LC, thereby reducing the efficiency of CTAB in controlling the LC alignment. The complexation also explains the absence of droplet formation with consequent turbidity of the continuous phase upon cooling when PVA is co-dissolved with $\mathrm{CTAB}$.

The deprotonation of PVA explains the relatively high conductivity of solutions with 5\% PVA shown in Figure 7, twice as high as a PVA-free CTAB solution of $15 \mathrm{mM}$ 
concentration. As CTAB is added to the PVA solution, the conductivity increases continuously, with a slope that is similar to the slope of a pure $\mathrm{CTAB}$ solution above the CMC. We attribute this to the complexation of PVA and CTAB into polymer-bound micelles.

Let us finally address the question of which side actually turns orthogonal in the cooling-induced hybrid-aligned shells in Figures 5 and S5. Since the CTAB is initially in the outer phase, its concentration should always be greater than that of the inner phase, even if the surfactant molecules can migrate through the shell membrane. Thus, it seems reasonable to assume that it is the outer shell interface that becomes orthogonally aligned when the shell adopts a hybrid director field configuration. This can obviously not be explained by surfactant migration through the shell, but rather by increased adsorption of $\mathrm{CTAB}$ onto the outer shell interface at low temperatures, suggesting that the PVA-CTAB interaction is also less favorable at $T<T_{\mathrm{K}}$. The respective temperature dependences of the interaction energies between PVA-CTAB and between $\mathrm{LC}-\mathrm{CTAB}$ might explain the reversible alignment transitions seen both in the first and in the second part of our study.

The easily observable change in texture of the LC shells as the solubility of $\mathrm{CTAB}$ in the outer phase is reduced turns the LC shells into reporters of the solution status. The potential of LC droplets as chemical and biological sensors has been well demonstrated by Abbott and co-workers. ${ }^{2,39,40}$ The present study suggests that LC shells may also be of interest in sensing contexts, in particular for detecting solutes that can penetrate into or through the shell, thereby changing the LC director alignment on the outside and/or inside, with a consequent change in the texture. The orders of magnitude larger diameter of shells compared to the micron-scale droplets that are optimal for biosensing would be beneficial from a read-out simplicity point of view, but the usefulness will critically depend on the minimum concentration of the analyte in the continuous phase for triggering the change in the internal phase, as well as the specificity of the response.

\section{CONCLUSIONS}

Our systematic study of nematic LC shells stabilized by different concentrations of $\mathrm{CTAB}$ on one side, at temperatures above and below $T_{\mathrm{K}}$, and by PVA on the other side, has demonstrated that the dramatic reduction in solubility of $\mathrm{CTAB}$ as the aqueous phase is cooled to $T<T_{\mathrm{K}}$ can be used to dynamically change the alignment of LC shells. Conversely, the asymmetrically prepared LC shells function as reporters of the reduced solubility on cooling (and, at appropriate CTAB concentrations, of the increased solubility on heating). A number of key observations allow us to formulate the hypothesis that the change is due to transport of CTAB through the LC into the originally surfactant-free aqueous PVA solution. This changes the LC director orientation on the side of the shell that is in contact with the latter solution, resulting in an easily detectable macroscopic texture change. The CTAB concentration required to see the effect increases by up to an order of magnitude if PVA is added to the CTAB solution at a concentration of $5 \mathrm{wt} \%$. This can be attributed to the documented strong interactions between PVA and CTAB when co-dissolved in water, leading to polymer-bound micelle complexes.

\section{ASSOCIATED CONTENT}

\section{S Supporting Information}

The Supporting Information is available free of charge on the ACS Publications website at DOI: 10.1021/acs.langmuir.9b00989.

Complementary microscopy images for 5CB shell behavior under additional representative conditions, including varying shell thickness, with $\mathrm{CTAB}$ in the inner and outer phases, respectively; representative microscopy images of E7 shell behavior with $\mathrm{CTAB}+$ PVA in the outer phase and PVA in the inner phase; macroscopic and microscopy images showing liquid crystal droplets in the continuous phase after cooling when $C T A B$ is used for shell stabilization (PDF)

\section{AUTHOR INFORMATION}

\section{Corresponding Author}

*E-mail: Jan.Lagerwall@lcsoftmatter.com. Phone: +352 4666 44 6219. Fax: +35246664436219.

ORCID

Venkata Subba Rao Jampani: 0000-0003-0881-2065

Jan P. F. Lagerwall: 0000-0001-9753-1147

Notes

The authors declare no competing financial interest.

\section{ACKNOWLEDGMENTS}

We thank C. Honorato Rios for help with the conductivity measurements, R. Kizhakidathazhath for fruitful discussions, and P. Dale, M. Beggiato, and S. Krishnamoorthy for additional experimental assistance. Financial support from the European Research Council under the European Union's Seventh Framework Programme (FP/2007-2013)/ERC Grant Agreement no. 648763 (consolidator project INTERACT) is gratefully acknowledged. A.S. acknowledges support from the Fonds National de la Recherche (FNR, doctoral training grant PRIDE MASSENA, grant code 2016/10935404). V.S.R.J. acknowledges financial support from the Fonds National de la Recherche (FNR, grant ID C17/MS/11703329/trendsetter).

\section{ADDITIONAL NOTE}

${ }^{a_{T}}$ The producer of our PVA, Sigma-Aldrich, reports $\mathrm{pH}$ of a $4 \%$ PVA solution to be in the range 4.5-6.5.

\section{REFERENCES}

(1) de Gennes, P.-G.; Prost, J. The Physics of Liquid Crystals; Clarendon Press: Oxford, U.K., 1993.

(2) Carlton, R. J.; Hunter, J. T.; Miller, D. S.; Abbasi, R.; Mushenheim, P. C.; Tan, L. N.; Abbott, N. L. Chemical and biological sensing using liquid crystals. Liq. Cryst. Rev. 2013, 1, 29-51.

(3) Urbanski, M.; Reyes, C. G.; Noh, J.; Sharma, A.; Geng, Y.; Jampani, V. S. R.; Lagerwall, J. P. Liquid crystals in micron-scale droplets, shells and fibers. J. Phys.: Condens. Matter 2017, 29, No. 133003.

(4) Lopez-Leon, T.; Fernandez-Nieves, A. Drops and shells of liquid crystal. Colloid Polym. Sci. 2011, 289, 345-359.

(5) Geng, Y.; Jang, J.-H.; Noh, K.-G.; Noh, J.; Lagerwall, J. P.; Park, S.-Y. Through the Spherical Looking-Glass: Asymmetry Enables Multicolored Internal Reflection in Cholesteric Liquid Crystal Shells. Adv. Opt. Mater. 2018, 6, No. 1700923.

(6) Noh, J.; Jampani, V. S. R.; Haba, O.; Yonetake, K.; Takezoe, H.; Lagerwall, J. P. Sub-second dynamic phototuning of alignment in azodendrimer-doped nematic liquid crystal shells. J. Mol. Liq. 2018, 267, 197-204. 
(7) Tran, L.; Lavrentovich, M. O.; Durey, G.; Darmon, A.; Haase, M. F.; Li, N.; Lee, D.; Stebe, K. J.; Kamien, R. D.; Lopez-Leon, T. Change in Stripes for Cholesteric Shells via Anchoring in Moderation. Phys. Rev. X 2017, 7, 167.

(8) Darmon, A.; Benzaquen, M.; Copar, S.; Dauchot, O.; LopezLeon, T. Topological defects in cholesteric liquid crystal shells. Soft Matter 2016, 12, 9280-9288.

(9) Darmon, A.; Dauchot, O.; Lopez-Leon, T.; Benzaquen, M. Elastic interactions between topological defects in chiral nematic shells. Phys. Rev. E 2016, 94, No. 062701.

(10) Zhou, Y.; Guo, A.; Zhang, R.; Armas-Perez, J.; MartínezGonzález, J.; Rahimi, M.; Sadati, M.; de Pablo, J. Mesoscale structure of chiral nematic shells. Soft Matter 2016, 12, 8983-8989.

(11) Manyuhina, O.; Bowick, M. Thick smectic shells. Int. J. NonLinear Mech. 2015, 75, 87-91.

(12) Seč, D.; Lopez-Leon, T.; Nobili, M.; Blanc, C.; FernandezNieves, A.; Ravnik, M.; Žumer, S. Defect trajectories in nematic shells: Role of elastic anisotropy and thickness heterogeneity. Phys. Rev. E 2012, 86, No. 020705(R).

(13) Liang, H.-L.; Zentel, R.; Rudquist, P.; Lagerwall, J. Towards tunable defect arrangements in smectic liquid crystal shells utilizing the nematic-smectic transition in hybrid-aligned geometries. Soft Matter 2012, 8, 5443-5450.

(14) Lopez-Leon, T.; Koning, V.; Devaiah, K. B. S.; Vitelli, V.; Fernandez-Nieves, A. Frustrated nematic order in spherical geometries. Nat. Phys. 2011, 7, 391-394.

(15) Fernández-Nieves, A.; Vitelli, V.; Utada, A.; Link, D. R.; Márquez, M.; Nelson, D. R.; Weitz, D. A. Novel defect structures in nematic liquid crystal shells. Phys. Rev. Lett. 2007, 99, No. 157801.

(16) Jampani, V. S. R.; Mulder, D. J.; De Sousa, K. R.; Gélébart, A.H.; Lagerwall, J. P. F.; Schenning, A. P. H. J. Micrometer-Scale Porous Buckling Shell Actuators Based on Liquid Crystal Networks. Adv. Funct. Mater. 2018, 28, No. 1801209.

(17) Schwartz, M.; Lenzini, G.; Geng, Y.; Rønne, P.; Ryan, P.; Lagerwall, J. Cholesteric Liquid Crystal Shells as Enabling Material for Information-Rich Design and Architecture. Adv. Mater. 2018, 30, No. 1707382.

(18) Lee, S.; Seo, H.; Kim, Y.; Kim, S. Structural Color Palettes of Core-Shell Photonic Ink Capsules Containing Cholesteric Liquid Crystals. Adv. Mater. 2017, 29, No. 1606894.

(19) Kim, J.-G.; Park, S.-Y. Photonic Spring-Like Shell Templated from Cholesteric Liquid Crystal Prepared by Microfluidics. Adv. Opt. Mater. 2017, 5, No. 1700243.

(20) Heo, I.-S.; Park, S.-Y. Smart shell membrane prepared by microfluidics with reactive nematic liquid crystal mixture. Sens. Actuators, B 2017, 251, 658-666.

(21) Akita, T.; Kouno, H.; Iwai, Y.; Uchida, Y.; Nishiyama, N. Room-temperature fabrication of mono-dispersed liquid crystalline shells with high viscosity and high melting points. J. Mater. Chem. C 2017, 5, 1303-1307.

(22) Kang, J.; Kim, S.; Fernandez-Nieves, A.; Reichmanis, E. Amplified Photon Upconversion by Photonic Shell of Cholesteric Liquid Crystals. J. Am. Chem. Soc. 2017, 139, 5708-5711.

(23) Noh, J.; Henx, B.; Lagerwall, J. P. Taming Liquid Crystal SelfAssembly: The Multifaceted Response of Nematic and Smectic Shells to Polymerization. Adv. Mater. 2016, 28, 10170-10174.

(24) Geng, Y.; Noh, J.; Drevensek-Olenik, I.; Rupp, R.; Lenzini, G.; Lagerwall, J. P. F. High-fidelity spherical cholesteric liquid crystal Bragg reflectors generating unclonable patterns for secure authentication. Sci. Rep. 2016, 6, No. 26840.

(25) Fleischmann, E.-K.; Liang, H.-L.; Kapernaum, N.; Giesselmann, F.; Lagerwall, J. P. F.; Zentel, R. One-piece micropumps from liquid crystalline core-shell particles. Nat. Commun. 2012, 3, No. 1178.

(26) Honaker, L.; Lagerwall, J.; Jampani, V. Microfluidic Tensiometry Technique for the Characterization of the Interfacial Tension between Immiscible Liquids. Langmuir 2018, 34, 24032409.
(27) Sharma, A.; Lagerwall, J. P. F. Influence of head group and chain length of surfactants used for stabilising liquid crystal shells. Liq. Cryst. 2018, 45, 2319-2328.

(28) Noh, J.; Reguengo De Sousa, K.; Lagerwall, J. P. F. Influence of interface stabilisers and surrounding aqueous phases on nematic liquid crystal shells. Soft Matter 2016, 12, 367-372.

(29) Brake, J.; Mezera, A.; Abbott, N. Effect of surfactant structure on the orientation of liquid crystals at aqueous-liquid crystal interfaces. Langmuir 2003, 19, 6436-6442.

(30) Brake, J.; Abbott, N. An experimental system for imaging the reversible adsorption of amphiphiles at aqueous-liquid crystal interfaces. Langmuir 2002, 18, 6101-6109.

(31) Dölle, S.; Lechner, B.-D.; Park, J. H.; Schymura, S.; Lagerwall, J. P. F.; Scalia, G. Utilizing the Krafft phenomenon to generate ideal micelle-free surfactant-stabilized nanoparticle suspensions. Angew. Chem., Int. Ed. 2012, 51, 3254-3257.

(32) Rosen, M. Surfactants and Interfacial Phenomena; John Wiley \& Sons, Inc.: Hoboken, 2004.

(33) Utada, A. S.; Lorenceau, E.; Link, D. R.; Kaplan, P. D.; Stone, H. A.; Weitz, D. A. Monodisperse double emulsions generated from a microcapillary device. Science 2005, 308, 537-541.

(34) Liang, H.-L.; Enz, E.; Scalia, G.; Lagerwall, J. Liquid Crystals in Novel Geometries prepared by Microfluidics and Electrospinning. Mol. Cryst. Liq. Cryst. 2011, 549, 69-77.

(35) Lopez-Leon, T.; Fernandez-Nieves, A. Topological transformations in bipolar shells of nematic liquid crystals. Phys. Rev. E 2009, 79, No. 021707.

(36) Peddireddy, K.; Kumar, P.; Thutupalli, S.; Herminghaus, S.; Bahr, C. Solubilization of thermotropic liquid crystal compounds in aqueous surfactant solutions. Langmuir 2012, 28, 12426-12431.

(37) Tadros, T. F. The interaction of cetyltrimethylammonium bromide and sodium dodecylbenzene sulfonate with polyvinyl alcohol. adsorption of the polymer-surfactant complexes on silica. J. Colloid Interface Sci. 1974, 46, 528-540.

(38) Goddard, E. D. Interactions of Surfactants with Polymers and Proteins; CRC Press, 2017.

(39) Manna, U.; Zayas-Gonzalez, Y.; Carlton, R.; Caruso, F.; Abbott, N.; Lynn, D. Liquid crystal chemical sensors that cells can wear. Angew. Chem., Int. Ed. 2013, 52, 14011-14015.

(40) Lin, I.-H.; Miller, D. S.; Bertics, P. J.; Murphy, C. J.; de Pablo, J. J.; Abbott, N. L. Endotoxin-Induced Structural Transformations in Liquid Crystalline Droplets. Science 2011, 332, 1297-1300. 